\title{
Simulation of the dynamic oscillatory response of electrorheological suspensions: Demonstration of a relaxation mechanism
}

\author{
D. J. Klingenberg \\ Department of Chemical Engineering and Rheology Research Center, \\ University of Wisconsin, Madison, Wisconsin 53706
}

(Received 1 June 1992; accepted 24 October 1992)

\begin{abstract}
Synopsis
The dynamic oscillatory response of electrorheological suspensions is investigated using a molecular dynamics-like simulation method, where suspensions are modeled as hard, monodis. perse, neutrally buoyant, dielectric spheres contained in a dielectric, Newtonian fluid between parallel-plate electrodes. The response is described by frequency-dependent moduli, which display a significant relaxation due to competition between hydrodynamic and electrostatic forces on spheres within thick clusters. For small amplitude deformation of monodisperse suspensions, the direct electrostatic contribution to the response obeys time-electric field strength superposition analogous to time-temperature superposition in polymer rheology. The response for monodisperse suspensions is dominated by a single relaxation time, in contrast to the broad dispersions commonly obscrved. Possible explanations for this discrepancy are discussed.
\end{abstract}

\section{INTRODUCTION}

Electrorheological (ER) suspensions, first studied extensively by Winslow (1949), display dramatic changes in rheological properties under large applied electric fields. Applications employing the electronically controlled, enhanced stress transfer capabilities of these suspensions are currently being developed. Discussions of applications and the present understanding of underlying phenomena can be found in recent reviews (Block and Kelly, 1988; Deinega and Vinogradov, 1984; Gast and Zukoski, 1989; Hartock et al., 1991; Jordan and Shaw, 1989).

Leading applications include ER engine mounts, shock absorbers, and other viscous damping devices. While progress has been made in understanding ER suspension behavior under steady shear, the above applications operate almost exclusively in dynamic or transient modes where understanding is poor. Experimental investigation of the dynamic response of ER suspensions has produced various results. For instance, moduli values are often reported to be sensitive to electric field strength (Gamato and Filisko, 1991; Xu and Liang, 1991), while other reports show them to be essentially independent of field strength (Korobko and Shulman, 1990; Shulman et al., 1989; Vinogradov et al., 1986; Yen and Achorn, 1991); responses are sometimes reported to be independent of strain frequency (Xu and Liang, 1991; Yen and Achorn, 1991), while in other situations moduli values increase or decrease with frequency (Gamato and Filisko, 1991; Korobko and Shulman, 1990; Otsubo et al., 1992; Shulman et al., 1989). Therc are currently no explanations for this diverse behavior, which prohibits utilization of ER technology to its full potential. 
Progress has been made in understanding the steady shear response and its relation to material properties. We have developed a molecular dynamics-like simulation method for investigating stress transfer in ER suspensions, which are modeled as neutrally buoyant, hard, monodisperse spheres subjected to hydrodynamic and electrostatic polarization forces (Klingenberg et al., 1989; Klingenberg et al., 1991a; Klingenberg et al., 1991b). This model successfully reproduces structure formation following electric field application, as well as many features of the steady-state rheological response, including: the dramatic change in rheological properties following the formation of fibrous structures; the existence of a static and dynamic yield stress; and saturation of the yield stress at large particle concentrations. The ability of this microscopic model to reproduce observed macroscopic properties suggests that it may be successfully extended to probe other unknown aspects of electrorheology.

In this paper, the dynamic simulation method described above is extended to investigate the dynamic oscillatory shear response of ER suspensions, with the goal of determining the microscopic features responsible for observed behavior. The dynamic simulation method is presented in the following section along with a discussion of the state of stress in ER suspensions. A significant result of the model chosen to represent ER suspensions is the property of time-field strength superposition. Simulation results are presented in Sec. III, where a stress relaxation mechanism resulting from hydrodynamic relaxation of electrostatic forces acting on spheres within clusters is described. The response for monodisperse suspensions is found to be dominated by a single relaxation time, in contrast to the broad dispersions commonly reported. In Sec. IV, several possible explanations for this discrepancy are briefly discussed. Specifically considered are distributions of cluster sizes, particle sizes and shapes, nonlinear effects, and more accurate treatments of the forces governing particle dynamics. Conclusions of this work are summarized in Sec. V.

\section{SIMULATION METHOD}

The method used to simulate ER suspension behavior has been described previously (Klingenberg et al., 1989; Klingenberg et al., 1991a). It is briefly reviewed here, and extended to include oscillatory flow.

An ER suspension is modeled as a system of neutrally buoyant, monodisperse spheres (diameter $\sigma$, dielectric constant $\epsilon_{p}$ ) in a Newtonian continuous phase (viscosity $\eta_{c}$, dielectric constant $\epsilon_{c}$ ). A sphere's motion is governed by the sum of electrostatic polarization forces induced by the applied electric field and hydrodynamic resistance. Electrostatic polarization forces are approximated in the point-dipole limit, and hydrodynamic forces are treated simply as Stokes' drag. In addition to these forces, a short-range repulsive force is added to represent hard-sphere behavior.

Ignoring inertia terms, the equation of motion in dimensionless form for sphere $i$ not near an electrode reduces to

$$
\frac{d \mathbf{r}_{i}^{*}}{d t^{*}}=\sum_{j \neq i} \mathbf{f}_{i j}^{\mathrm{el} *}+\sum_{j} \mathbf{f}_{i j}^{\mathrm{el} *}+\sum_{j \neq i}^{\prime \prime} \mathbf{f}_{i j}^{\mathrm{rep} *}+\mathbf{u}^{\infty}\left(\mathbf{r}_{i}^{*}\right),
$$

where the asterisks denote dimensionless quantities. The length, force, and time scales are given by

$$
l_{s}=\sigma, \quad F_{s}=\frac{3}{16} \pi \epsilon_{0} \epsilon_{c} \sigma^{2} \beta^{2} E_{0}^{2}, \quad t_{s}=3 \pi \eta_{c} \sigma^{2} / F_{s}=16 \eta_{\mathcal{d}} / \epsilon_{0} \epsilon_{c} \beta^{2} E_{0}^{2}
$$



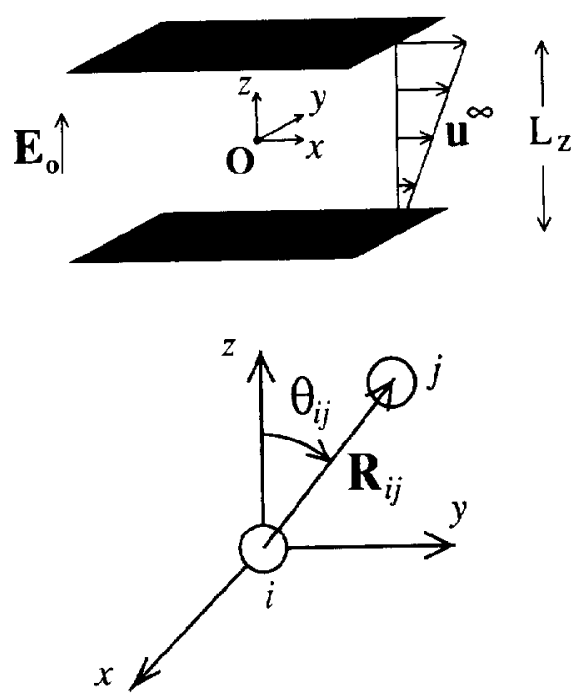

FIG. 1. Schematic diagrams showing the geometries of the sheared suspension and sphere pairs. The bottom electrode is held fixed and the top electrode is displaced in the $x$ direction. $\mathbf{R}_{i j}$ is the center-to-center separation and $\theta_{i j}$ is the angle between the line-of-centers and the applied electric field.

$F_{s}$ is the magnitude of the electrostatic interaction and $t_{s}$ corresponds to the time required for a sphere to move its diameter under a constant applied force $F_{s}$.

The terms on the right side of Eq. (1) represent forces acting on the sphere centers: $\mathbf{f}_{i j}^{\mathrm{el} *}$ is the pair electrostatic force and $\mathbf{f}_{i j}^{\mathrm{rep} *}$ is the short-range repulsive force, each referring to the force on sphere $i$ at the center of a spherical coordinate system due to sphere $j$ at $\left(r_{i j}^{*}, \theta_{i j}\right)$ (ER suspension geometry is depicted in Fig. 1):

$$
\begin{gathered}
\mathbf{f}_{i j}^{\mathrm{el} *}\left(r_{i j}^{*} \theta_{i j}\right)=\left(\frac{1}{r_{i j}^{*}}\right)^{4}\left[\left(3 \cos ^{2} \theta_{i j}-1\right) \mathbf{e}_{r}+\sin 2 \theta_{i j} \mathbf{e}_{\theta}\right], \\
\mathbf{f}_{i j}^{\mathrm{rep} *}\left(r_{i j}^{*}\right)= \begin{cases}\exp \left[-\left(r_{i j}^{*}-1\right) / 0.01\right]\left(-\mathbf{e}_{r}\right) & j=\text { sphere, } \\
\exp \left[-\left(h_{i}^{*}-0.5\right) / 0.01\right] \mathbf{n} & j=\text { electrode. }\end{cases}
\end{gathered}
$$

Here, $h_{i}^{*}=L_{z}^{*} / 2-\left|z_{i}^{*}\right|$ is the dimensionless distance between the sphere center and the electrode, and $\mathbf{n}$ is the unit normal directed into the fluid. The prime in the second summation in Eq. (1) indicates the electrostatic force between sphere $i$ and the images of sphere $j$ reflected orientation intact about the electrode surfaces; these forces are summed over all images including those of sphere $i$. The double prime on the third summation indicates repulsive forces are summed over all other spheres and the electrodes.

The last term in Eq. (1) represents the hydrodynamic drag on sphere $i$ due to the ambient flow at $\mathbf{r}_{i}^{*}$. For oscillatory shear flow, where the local strain is $\gamma\left(t^{*}\right)$ $=\gamma_{0} \sin \omega^{*} t^{*}$, the ambient fluid velocity is given by

$$
\mathbf{u}^{\infty *}\left(\mathbf{r}_{i}^{*}\right)=\omega^{*} \gamma_{0}\left(z_{i}^{*}+L_{z}^{*} / 2\right) \cos \omega^{*} t^{*} \mathbf{e}_{x}
$$

Equation (1) governs the motion of spheres not near an electrode surface. As discussed by Klingenberg et al. (1991a), a sphere whose surface is within $\delta_{w}$ 
$(=0.05 \sigma)$ of an electrode surface is "stuck" and assumes the lateral velocity of the electrode. The $z$ component of the motion is assumed unaffected by the sphere being "stuck," making the scalar equations of motion for such a sphere

$$
\begin{gathered}
\frac{d x_{i}^{*}}{d t^{*}}=u_{\mathrm{elec}}^{*}, \\
\frac{d y_{i}^{*}}{d t^{*}}=0, \\
\frac{d z_{i}^{*}}{d t^{*}}=\sum_{j \neq i} f_{z, i j}^{\mathrm{el} *}+\sum_{j} f_{z, i j}^{\mathrm{el} *}+\sum_{j \neq i}^{\prime \prime} f_{z, i j}^{\mathrm{ep} *},
\end{gathered}
$$

where the fact that $u_{y}^{\infty}=u_{z}^{\infty}=0$ in simple shear has been used. $u_{\text {elec }}^{*}$ is given by

$$
u_{\mathrm{elec}}^{*}= \begin{cases}\dot{\gamma}^{*} L_{z}^{*} & \text { top electrode, } \\ 0 & \text { bottom electrode. }\end{cases}
$$

This sticking condition is based on experimental observation (Klingenberg and $\mathrm{Zu}$ koski, 1990), and is required to obtain deformation in the limit of zero frequency (Klingenberg et al., 1991a).

For a given configuration, the dynamic oscillatory response at large times (i.e., after transient responses have decayed) depends only on $\gamma_{0}$ and $\omega^{*}$. In this paper, only the small amplitude shear response is considered, typically $\gamma_{0}=10^{-4}$. In this case, the response is linear and can be represented by frequency-dependent storage and loss components of the shear modulus $G^{*}\left(t^{*}\right)$ :

$$
G^{*}\left(t^{*}\right)=\frac{\sigma_{x z}^{*}\left(t^{*}\right)}{\gamma_{0}}=G^{*}\left(\omega^{*}\right) \sin \omega^{*} t^{*}+G^{\prime *}\left(\omega^{*}\right) \cos \omega^{*} t^{*},
$$

where $\sigma_{x z}^{*}\left(t^{*}\right)$ is the time-dependent shear stress acting in the $x$ direction on a plane normal to the $z$ direction. The goal of this work is to determine the features that influence the storage and loss moduli, $G^{*}\left(\omega^{*}\right)$ and $G^{\prime *}\left(\omega^{*}\right)$.

In general, the total, instantaneous stress is calculated from (Batchelor, 1970, 1977)

$$
\boldsymbol{\sigma}^{\text {total }}=(\mathrm{IT})+2 \eta_{c} \mathbf{E}^{\infty}+\frac{1}{V} \sum_{i=1}^{N}\left(\mathbf{S}_{i}^{H}+\mathbf{S}_{i}^{P}\right)-\frac{1}{V} \sum_{i=1}^{N} \mathbf{r}_{i} \mathbf{F}_{i}^{\text {total }} .
$$

(IT) represents an isotropic term of no interest, $\mathrm{E}^{\infty}$ is the macroscopic rate of strain tensor $\left(\left|\mathbf{E}^{\infty}\right| \propto \omega \gamma_{0}\right), \Sigma\left(\mathbf{S}_{i}^{H}+\mathbf{S}_{i}^{P}\right)$ represents contributions from hydrodynamic forces, $-\Sigma \mathbf{r}_{i} \mathbf{F}_{i}^{\text {total }}$ represents the nonhydrodynamic contribution arising from particle interactions (electrostatic and short-range repulsive forces), and $V$ is the suspension volume. The stress represented by Eq. (10) is conveniently expressed as a sum of direct electrostatic (plus repulsive) and hydrodynamic contributions,

$$
\boldsymbol{\sigma}^{\text {total }}=\sigma^{E}+\sigma^{H},
$$

where

$$
\sigma^{E}=\frac{-1}{V} \sum_{i=1}^{N} \mathbf{r}_{i} \mathbf{F}_{i}^{\mathrm{total}}
$$




$$
\begin{aligned}
\sigma^{H} & =2 \eta_{c} \mathbf{E}^{\infty}+\frac{1}{V} \sum_{i=1}^{N}\left(\mathbf{S}_{i}^{H}+\mathbf{S}_{i}^{P}\right), \\
& =2 \eta_{c} \mathbf{E}^{\infty}+\frac{1}{V} \sum_{i=1}^{N} \int_{A_{i}}\left[\left(\mathbf{r}-\mathbf{r}_{i}\right) \boldsymbol{\sigma} \cdot \mathbf{n}-\frac{1}{3} \delta\left(\mathbf{r}-\mathbf{r}_{i}\right) \cdot \boldsymbol{\sigma} \cdot \mathbf{n}\right] d A
\end{aligned}
$$

Here $\sigma$ is the hydrodynamic stress due to both the applied straining flow plus the flow resulting from interparticle forces, $\mathbf{r}_{i}$ designates the center of the $i$ th sphere, $\delta$ is the unit tensor, and $d A$ is a surface area element on sphere $i$ with unit normal $\mathbf{n}$ directed into the continuous phase. Provided the volume containing the particles is sufficiently large, the stress in Eq. (10) may be equated with the ensemble-averaged, macroscopic stress. The distinction between electrostatic and hydrodynamic contributions is not unique. The above separation is chosen because the hydrodynamic contribution is directly proportional to the solvent viscosity and hence vanishes as the deformation rate vanishes or when the solvent is removed, whereas the electrostatic contribution remains in both situations. Note that the direct contributions $\sigma^{E}$ and $\sigma^{H}$ are not independent as both electrostatic and hydrodynamic forces influence structure and hence the summations in Eqs. (12) and (13) (Klingenberg et al., 1991c).

The dimensionless electrostatic shear stress is calculated in the present framework as

$$
\sigma_{x z}^{E_{*}}=-\frac{1}{V^{*}} \sum_{i=1}^{N} z_{i}^{*} f_{x i}^{\text {total }}
$$

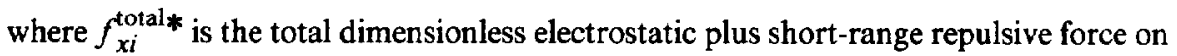
sphere $i$ acting in the $x$ direction. This stress corresponds to direct electrostatic contributions to the moduli.

$$
G^{E *}\left(t^{*}\right)=\frac{\sigma_{x z}^{E *}\left(t^{*}\right)}{\gamma_{0}}=G^{E^{*}}\left(\omega^{*}\right) \sin \omega^{*} t^{*}+G^{E^{\prime *}}\left(\omega^{*}\right) \cos \omega^{*} t^{*}
$$

From here forward, only the electrostatic contribution to the stress is considered unless specifically stated otherwise. Hence, the superscript " $E$ " will be omitted unless it is necessary to avoid confusion.

A significant consequence of this model is that the electrostatic stress and moduli possess the property of time-field strength superposition, analogous to timetemperature superposition in polymer rheology (Ferry, 1980). The moduli, scaled with the electrostatic stress scale $\sigma_{s}=(3 / 16) \pi \epsilon_{0} \epsilon_{c} \beta^{2} E_{0}^{2}$, depend only on the dimensionless frequency, $\omega^{*}=\omega 16 \eta_{c} / \epsilon_{0} \epsilon_{c} \beta^{2} E_{0}^{2}$, for a particular configuration. Thus the field strength dependences of the direct electrostatic moduli are determined from both the explicit scaling and the dimensionless frequency dependence [i.e., $G^{\prime}=E_{0}^{2} f_{1}(\omega /$ $\left.E_{0}^{2}\right), G^{\prime \prime \prime}=E_{0}^{2} f_{2}\left(\omega / E_{0}^{2}\right)$ ]. Another important implication is if experimental frequencies are only accessible over a small range, dimensionless frequencies can be sampled over a much larger range by varying the electric field strength. For instance, large dimensionless frequencies are obtained by using either large frequencies or small field strengths; small dimensionless frequencies are obtained at small frequencies or large field strengths. Note that for the typical parameter values $\eta_{c}=0.050 \mathrm{~Pa} \mathrm{~s}, \epsilon_{c}=2.0$, 


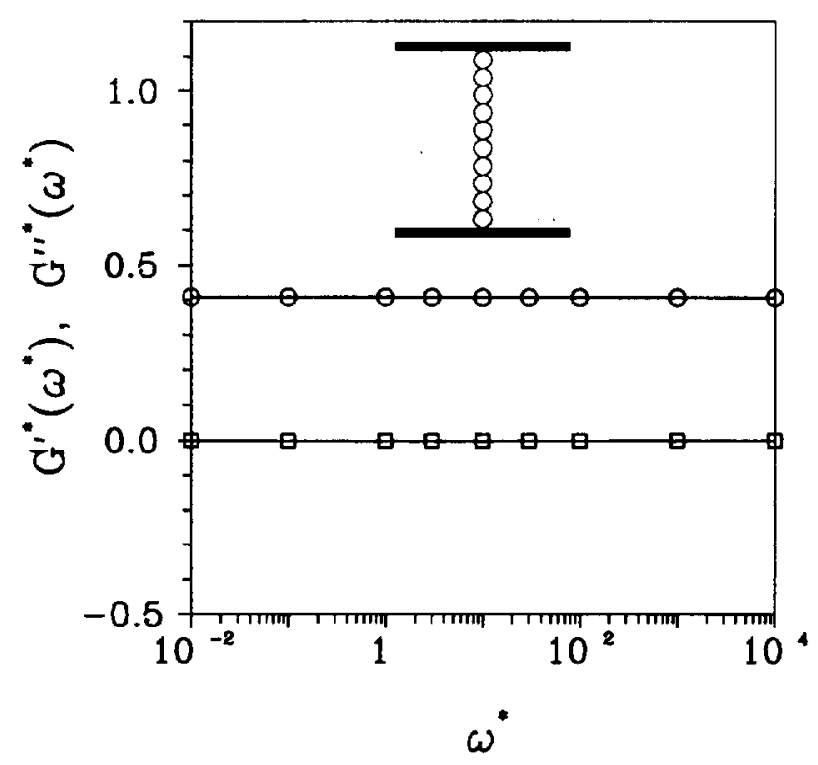

FIG. 2. Dynamic oscillatory response for a single-sphere-width chain spanning the electrode gap. (O)$G^{*}\left(\omega^{*}\right) ;(\square)-G^{\prime \prime}\left(\omega^{*}\right)$

$\beta=0.50$, and $E_{0}=10^{6} \mathrm{~V} / \mathrm{m}$, a dimensionless frequency of $\omega^{*}=1$ corresponds to a frequency of $\omega=5.5 \mathrm{~s}^{-1}$. Finally, this frequency scaling is analogous to the Mason number scaling of the steady shear response reported by Marshall et al. (1989) (Mn $\left.=\eta_{c} \dot{\gamma} / 2 \epsilon_{0} \epsilon_{d} B^{2} E_{0}^{2}\right)$. In fact, replacing the steady shear rate, $\dot{\gamma}$, with the angular frequency, $\omega$, one finds $\omega^{*}=32 \times M n$.

Simulations are performed by placing $N$ spheres in a two-dimensional "cell," with periodic boundaries in the $x$ direction located at $x^{*}= \pm L_{x}^{*} / 2$ and electrodes at $z^{*}=$ $\pm L_{z}^{*} / 2$ (three-dimensional simulations are considered in Sec. IV). The equation of motion for each sphere is integrated numerically using Euler's method with time step $\Delta t^{*} \leqslant 10^{-3}$. Forces are evaluated only within a cutoff radius $r_{c}^{*}=L_{z}^{*} / 2$. Results are insensitive to decreases in the time step magnitude and increases in the cutoff radius. Simulations under oscillatory flow are performed after a metastable structure under no flow is obtained, either by manufacture or by integration of the equation of motion until motion ceases. Moduli values are determined by Eq. (16), using stress values obtained after decay of all transient responses. Linear responses are obtained for all situations reported here, as determined by the ability of Eq. (16) to describe simulated stresses to at least four significant figures. For structures formed from random configurations, a constant, residual stress must be subtracted from the calculated stress since static metastable structures invariably possess a nonzero stress (for finite systems).

\section{RESULTS: DEMONSTRATION OF A RELAXATION MECHANISM}

\section{A. Response for single-sphere-width strands}

Consider first the response for a linear strand of spheres aligned with the electric field, connecting the electrodes $\left(N=10, L_{x}^{*}=10.0, L_{z}^{*}=10.0\right.$; see Fig. 2$)$. For this structure, the storage modulus $G^{*}\left(\omega^{*}\right)$ is essentially independent of frequency. The 


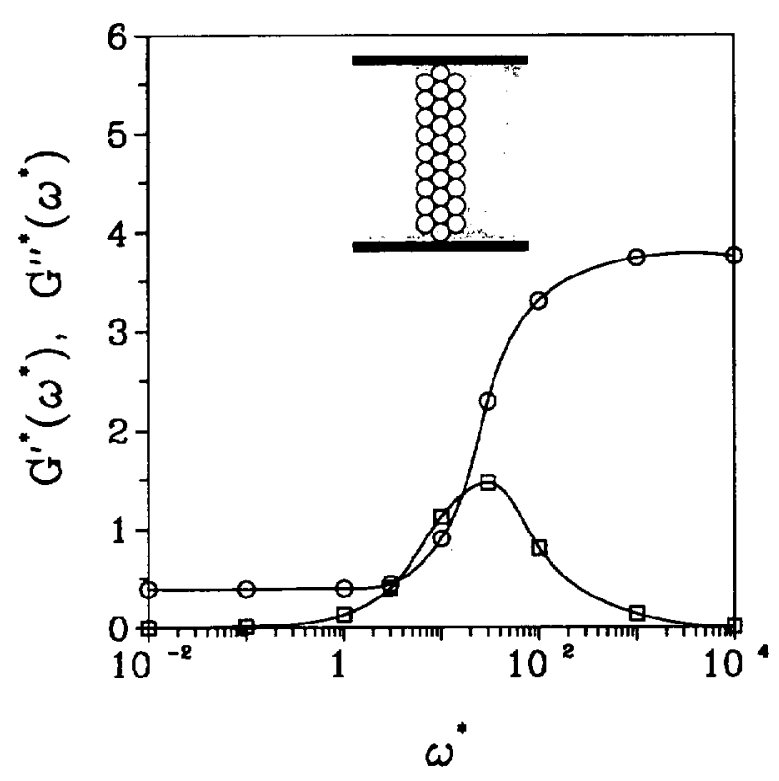

FIG. 3. Dynamic oscillatory response for a thick, 28-sphere cluster. The solid lines are cubic spline interpolations between the simulation data points. $(O)-G^{\prime *}\left(\omega^{*}\right) ;(\square)-G^{\prime \prime *}\left(\omega^{*}\right)$.

loss modulus, $G^{\prime *}\left(\omega^{*}\right)$, is negative but negligible; the magnitude of the loss tangent, $\left|\tan \delta\left(\omega^{*}\right)\right|=\left|G^{\prime \prime *}\left(\omega^{*}\right) / G^{*}\left(\omega^{*}\right)\right| \leqslant 10^{-3}$ for all frequencies (see Fig. 2). This simple response is due to affine sphere motion-each sphere is displaced in concert with the ambient shear flow at all frequencies. Hence there is no hydrodynamic resistance and no mechanism for relaxation. Since suspension structure is independent of frequency, the electrostatic shear stress [Eq. (15)], and hence the moduli, are independent of frequency. (It is noted that there is a negligible relaxation associated with the slight nonaffine motion of the spheres attached to an electrode. These spheres are forced to translate with th e electrode, while the ambient fluid displacement at the sphere center differs slightly. This effect causes sphere motion to slightly lead the applied strain, producing the negligible, negative loss modulus.)

\section{B. Response for a thick cluster}

Consider next the response for a thick cluster, composed of the previous singlesphere-width strand with two additional strands placed along each side of the original strand $\left(N=28, L_{x}^{*}=10.0, L_{z}^{*}=10.0\right.$; see Fig. 3). The frequency-dependent moduli for this structure are presented in Fig. 3. The loss modulus scales as $G^{\prime \prime}\left(\omega^{*}\right) \sim \omega^{*}$ at small frequencies and as $G^{\prime \prime *}\left(\omega^{*}\right) \sim \omega^{*-1}$ at large frequencies, passing through a maximum at a dimensionless frequency of $\omega^{*} \approx 25$. Near this frequency, the storage modulus increases from a small frequency plateau value, $G^{*}(0)$, to a large frequency plateau value, $G^{\prime *}(\infty)$. Simulations performed at $\gamma_{0}=10^{-3}$ give identical moduli values, indicating a linear response for $\gamma_{0} \leqslant 10^{-3}$ (and perhaps larger values).

This thick cluster exhibits a significant relaxation whereas the single-sphere-width strand exhibits none. Relaxation results from the addition of the side chains, as this is the only difference between the thin and thick clusters. The relaxation mechanism 


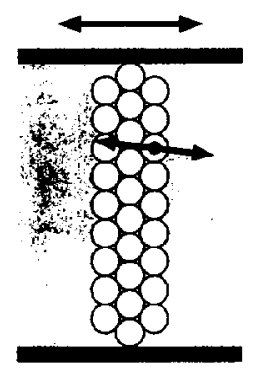

$\omega^{*}<1$

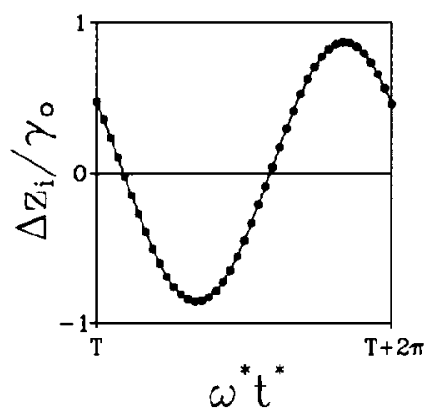

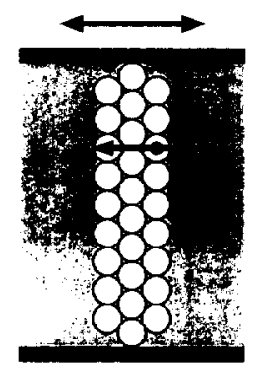

$\omega^{*} \gg 1$

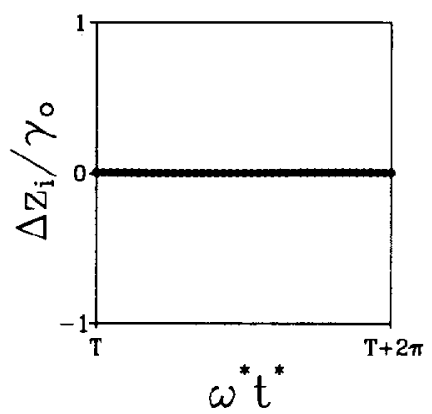

FIG. 4. Illustration of the dynamic structures in the small and large frequency limits for the 28-sphere cluster. The arrows represent the displacement directions of the electrodes and a particular sphere in the right side chain. The corresponding plots show the $z$ displacement magnitudes of the indicated sphere. obtained from the simulation. Note that displacement magnitudes are $\mathscr{C}\left(10^{-4}\right)$.

becomes apparent when the motions of individual spheres and the overall deformation of the cluster are considered in the small and large frequency limits.

At very small frequencies, deformation is completely determined by the displacement of the spheres stuck to the electrodes-hydrodynamic resistance to sphere motion is insignificant at these small deformation rates. As a result, the net electrostaticplus-repulsive force on each sphere not attached to an electrode is zero at every instant [Eq. (1)]. The extensional and rotational components of the quasi-static deformation require that spheres in the stress-bearing center strand deform affinely (with a slight correction described in Sec. III A), while sphere displacement in the side chains possesses components in both the $x$ and $z$ directions. This motion is depicted in Fig. 4 , where the displacement directions of a particular sphere in the right side chain are shown. Also presented in Fig. 4 is a plot of the $z$ component of the displacement divided by the strain amplitude as a function of time for the sphere indicated (obtained from simulations at $\omega^{*}=10^{-2}$ ).

At large frequencies, the motion of spheres in the side chains is significantly different. Here, hydrodynamic resistance controls the displacement of each sphere. At any particular instant, there is still a net electrostatic-plus-repulsive force component in the $z$ direction, but there is insufficient time available during a half period for the sphere to move in that direction. This time, proportional to $1 / \omega^{*}$, vanishes as $\omega^{*} \rightarrow \infty$. As a result, each sphere displaces only in the $x$ direction. This situation is also depicted in Fig. 4, where the displacement directions of a particular sphere at large frequencies 
is shown, along with a plot of its displacement in the $z$ direction as a function of time (obtained from simulations at $\omega^{*}=10^{4}$ ). Motion is affine in this large frequency limit. However, note that this is only true for spheres interacting through continuous repulsive forces-for two hard spheres in contact with different $z$ coordinates, at least one must be moving relative to the ambient shear flow.

Thus the dispersion in Fig. 3 is due to hydrodynamic relaxation of electrostatically driven sphere motion, providing a frequency-dependent dynamic structure. As the shear stress is simply a summation over position-dependent pair interactions, the stress and hence the moduli will also be frequency dependent. The critical frequency marking the loss modulus maximum is directly related to the characteristic relaxation time, which in this case is just a fraction of the time scale $t_{s}$. The peak in $G^{\prime \prime *}$ is pronounced because each sphere sees essentially the same environment, and therefore the response is dominated by a single relaxation time (factors that broaden the dispersion are considered in the following section ).

Although similar in some respects, this hydrodynamic relaxation mechanism is significantly different from that proposed by McLcish et al. (1991), who attributed relaxation to the motion of "free strings" ( single-sphere-width chains attached to at most one electrode). In their theory, free strings deform affinely at large frequencies, but are able to relax at small frequencies due to the electrostatic forces, thus providing a relaxation mechanism. This mechanism is only likely to operate at small concentrations where thin, unattached chains are likely to exist. In contrast, the mechanism presented in this paper occurs within thicker clusters and hence will be active especially at moderate to large concentrations. It is significant, however, that both mechanisms arise from the electrostatic polarization model for ER suspensions and do not rely on fiber rupture-dissipation is realized at infinitesimal as well as finite strain amplitudes. It is also important to note that relaxation of free strings is captured by the present model-however, only relaxation of thick clusters typically found in concentrated ER suspensions is considered in this paper.

\section{DISCUSSION}

Most experimental data reported in the literalure show a broad dispersion-storage and loss moduli are often reported to be insensitive to frequency over a large range, often several decades. These results are quite different from the simulation results presented above for a simple, uniform suspension structure. Below, several possible mechanisms for dispersion broadening are considered: varying cluster sizes and size distributions, artifacts associated with two-dimensional systems, varying particle sizes and size distributions, particle shape distributions, and nonlinear effects. In Sec. IV E, effects expected from more accurate treatments of electrostatic and hydrodynamic forces are briefly discussed.

\section{A. Variation with cluster size and size distribution}

The dramatic change in the relaxation spectrum in going from a single-spherewidth strand to a thick cluster suggests the dispersion width ought to depend on the cluster size and cluster size distribution. To investigation the influence of cluster size, simulations were performed using a cluster larger than that depicted in Fig. $3(N=44$, $L_{x}^{*}=10, L_{z}^{*}=10$; see Fig. 5). The results are shown in Fig. 5. Although the moduli values are larger than those in Fig. 3, and the peak in $G^{\prime \prime}\left(\omega^{*}\right)$ is shifted to smaller frequency, there is no qualitative difference. The shift to smaller frequency is due to the larger excursions in the $z$ direction of spheres in the outer side chains during 


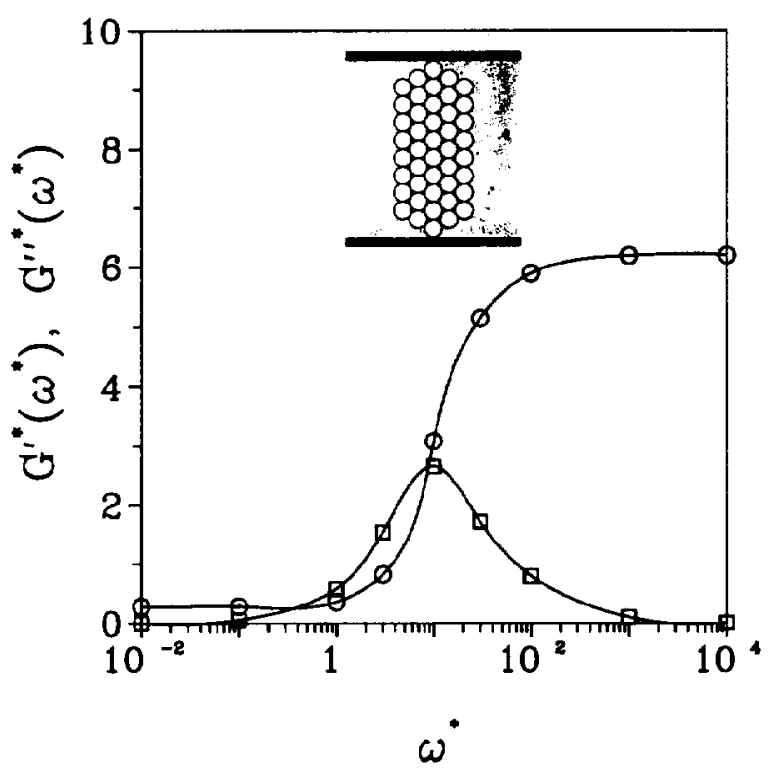

FIG. 5. Dynamic oscillatory response for a thick, 44-sphere cluster. The solid lines are cubic spline interpolations between the simulation data points. $(O)-G^{*}\left(\omega^{*}\right)$; $(\square)-G^{\prime *}\left(\omega^{*}\right)$.

oscillation. At a given frequency, the hydrodynamic resistance on these spheres is larger than on spheres in the side chains in Fig. 3, resulting in restricted motion at smaller frequencies.

The effect of distributions of cluster sizes and shapes was probed by performing simulations on systems obtained from random structures. 250 spheres were placed randomly in a simulation cell of dimensions $\left(L_{x}^{*}, L_{z}^{*}\right)=(50,10)$. A fibrous structure was formed by integrating the equations of motion with $\mathbf{u}^{\infty *}=0$ until motion ceased. A second structure was obtained by shearing this structure at a dimensionless steady shear rate of $j^{*}=10^{-3}$ to a strain of 4.0 , followed by relaxation to a metastable structure [see Klingenberg et al. (1991a) for a description of simulations under steady shear and relaxation]. The resulting structures are presented in Figs. 6(a) and 6(b), along with the corresponding moduli. The $G^{\prime \prime *}\left(\omega^{*}\right)$ curves do become broader due to distributions of clusters of various sizes and shapes, including several free strings, but there are still pronounced peaks. It is therefore expected that other features besides distributions of cluster sizes and shapes are required to produce broad dispersions.

\section{B. Results in three dimensions}

Simulations were also performed on a three-dimensional system similar in structure to the two-dimensional cluster described in Fig. 3. 25 spheres were placed in simulation cell, dimensions $\left(L_{x}^{*}, L_{y}^{*}, L_{z}^{*}\right)=(5,5,5)$, with periodic boundaries in the $x$ and $y$ directions. The simulation results are shown in Fig. 7. The qualitative aspects of the relaxation spectrum are similar to the results for the two-dimensional clusters-a sharp peak in the loss modulus at a critical frequency where the storage modulus increases from its low-frequency plateau value to its high-frequency value. Again, the loss modulus scales as $G^{\prime \prime *}\left(\omega^{*}\right) \sim \omega^{*}$ at small frequencies, and $G^{\prime \prime *}\left(\omega^{*}\right) \sim \omega^{*-1}$ at 

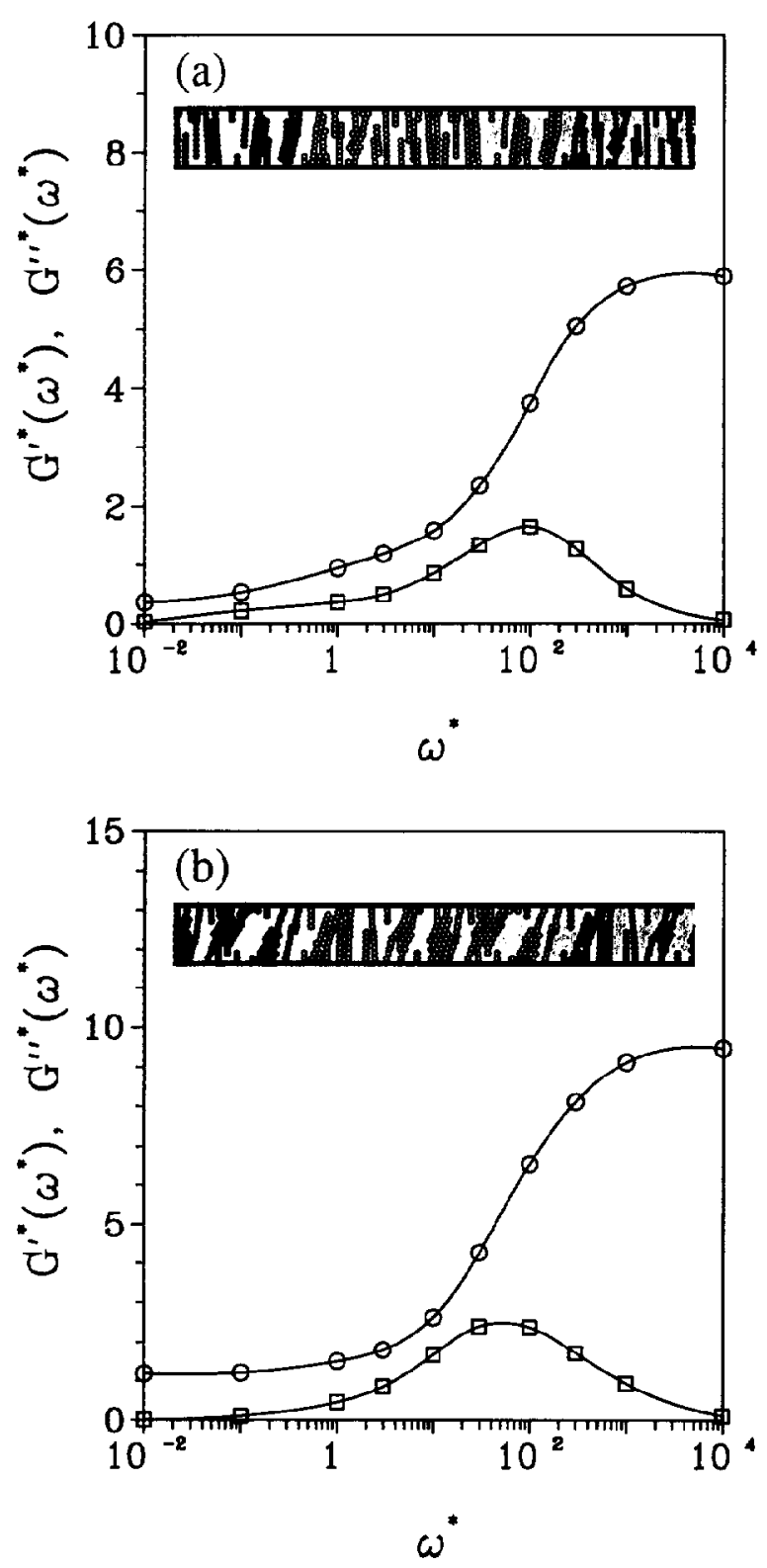

FIG. 6. Dynamic oscillatory response for 250 -sphere structures. The filled spheres represent periodic images of the open spheres. The solid lines are cubic spline interpolations between the simulation data points. (a) Structure obtained from static formation from a random configuration; (b) structure obtained by shearing the previous structure at a dimensionless shear rate of $10^{-3}$ to a strain of 4.0 , followed by relaxation to a metastable configuration. (O) $-G^{* *}\left(\omega^{*}\right) ;(\square)-G^{\prime \prime *}\left(\omega^{*}\right)$.

large frequencies. Hence, the absence of a broad peak in $G^{\prime \prime *}\left(\omega^{*}\right)$ in two dimensions is not due to an artifact associated with system dimension.

\section{Variation with particle size and size distribution}

The relaxation mechanism presented in Sec. III demonstrates a response independent of particle size for monodisperse suspensions. The explanation is as follows: the 


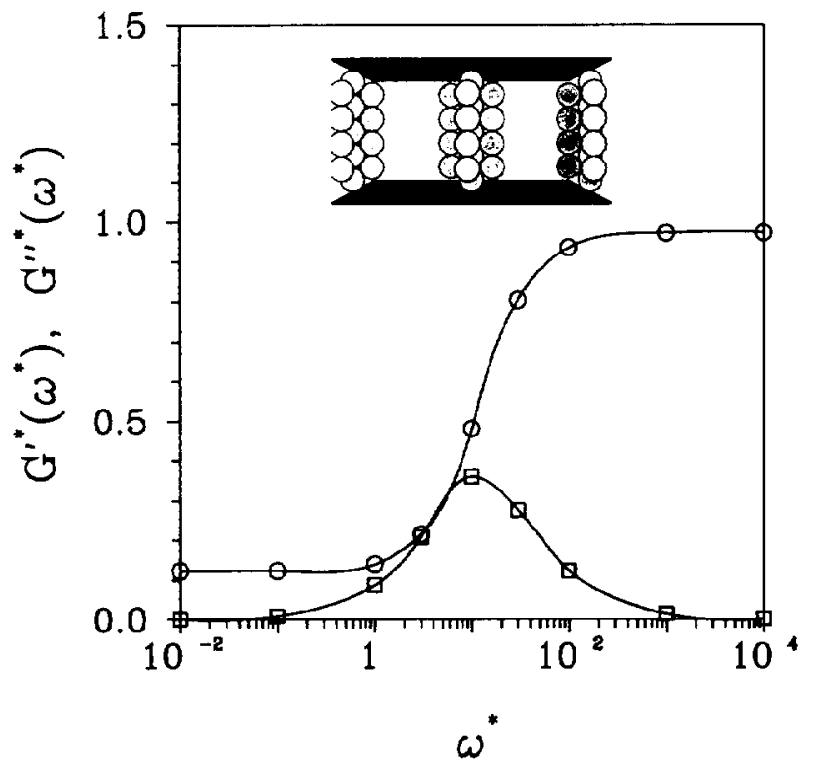

FIG. 7. Dynamic oscillatory response for 25 -sphere, three-dimensional cluster (the three-dimensional illustration contains periodic images of some spheres). The solid lines are cubic spline interpolations between the simulation data points. (O)-G* $G^{*}\left(\omega^{*}\right) ;(\square)-G^{\prime *}\left(\omega^{*}\right)$.

characteristic relaxation time, $\tau$, is equal to the time required for a sphere to move a fraction of its diameter due to a force $F_{s}$. Balancing the magnitudes of hydrodynamic and electrostatic forces, the relaxation time is found to be proportional to the time scale $t_{s}$ :

$$
\begin{gathered}
F^{H} \approx F^{E}, \\
3 \pi \eta_{c} \sigma \frac{\sigma}{\tau} \approx \frac{3}{16} \pi \epsilon_{0} \epsilon_{c} \sigma^{2} \beta^{2} E_{0}^{2}, \\
\Rightarrow \tau \approx \frac{16 \eta_{c}}{\epsilon_{0} \epsilon_{c} \beta^{2} E_{0}^{2}} .
\end{gathered}
$$

The relaxation time is independent of particle size for monodisperse suspensions due to canceling of equivalent particle size dependencies in the hydrodynamic and electrostatic forces.

A significantly different result is obtained, however, when polydisperse suspensions are considered. The clectrostatic force on sphere $i$ (diameter $\sigma_{i}$ ) at the origin due to sphere $j$ (diameter $\left.\sigma_{j}\right)$ at $\left(R_{i j}, \theta_{i j}\right)$ is given in the point-dipole limit by

$$
\mathbf{F}_{i j}^{\mathrm{el}}\left(R_{i j}, \theta_{i j}\right)=F_{s}\left(\sigma_{i}, \sigma_{j}\right)\left(\frac{R_{\min }}{R_{i j}}\right)^{4}\left[\left(3 \cos ^{2} \theta_{i j}-1\right) \mathbf{e}_{r}+\sin 2 \theta_{i j} \mathbf{e}_{\theta}\right],
$$

where $R_{\min }=\left(\sigma_{i}+\sigma_{j}\right) / 2$. The electrostatic force scale is now a sensitive function of both particle sizes, 


$$
\begin{gathered}
F_{s}\left(\sigma_{i}, \sigma_{j}\right)=\frac{3}{16} \pi \epsilon_{0} \epsilon_{c} \beta^{2} E_{0}^{2}\left[\sigma_{j}^{2} \frac{16 \lambda_{i j}^{3}}{\left(1+\lambda_{i j}\right)^{4}}\right], \\
\lambda_{i j}=\sigma_{i} / \sigma_{j}
\end{gathered}
$$

Note the electrostatic force goes to zero as $\lambda_{i j} \rightarrow 0$ and $\lambda_{i j} \rightarrow \infty$. As a result of this sensitive dependence of the force on the particle sizes, balancing electrostatic and hydrodynamic forces now gives a relaxation time that depends on the sizes of the two spheres under consideration. Retaining Stokes' law scaling for the hydrodynamic force on sphere $i$,

$$
F_{i}^{H} \approx 3 \pi \eta_{c} \sigma_{i}^{2} / \tau_{i j}
$$

a relaxation time for sphere $i$ due to sphere $j$ is obtained:

$$
\tau_{i j}=\frac{16 \eta_{c}}{\epsilon_{0} \epsilon_{c} \beta^{2} E_{0}^{2}}\left[\frac{\left(1+\lambda_{i j}\right)^{4}}{16 \lambda_{i j}}\right]
$$

The divergence in the relaxation time as $\lambda_{i j} \rightarrow 0$ or $\infty$ is due to the pair electrostatic force vanishing while hydrodynamic resistance remains finite for finite velocities. Significant effects resulting from polydispersity are expected. First, for spheres of different sizes, motion will relax with larger relaxation times than for equal-sized spheres, indicating a shift of the oscillatory response to smaller frequencies. Second, one must be careful when applying the concept of time-field strength superposition since the proper time scale remains undetermined for polydisperse suspensions. Third, since polydisperse suspensions possess a distribution of sphere-pair diameter ratios, the dispersions of $G^{\prime *}\left(\omega^{*}\right)$ and $G^{\prime \prime *}\left(\omega^{*}\right)$ are expected to broaden significantly. It is noted that for the experiments reporting broad dispersion, the suspensions are commonly polydisperse. However, in a recent article, Otsubo et al. (1992) reported dynamic oscillatory ER data for monodisperse silica suspensions. They found that for small amplitude deformation, the frequency dependencies of the moduli are well characterized by a single relaxation time-the loss modulus as a function of frequency demonstrated a pronounced maximum. While existing experimental data are consistent with the prediction of polydispersity broadening moduli dispersion, conclusive experimental verification is lacking.

Finally we note the possibility that moduli magnitudes may be affected by polydispersity. As pointed out above, the magnitude of the pair electrostatic interaction scales as $\epsilon_{0} \epsilon_{c} \beta^{2} E^{2} \times \sigma_{j}^{2} 16 \lambda_{i j}^{3} /\left(1+\lambda_{i j}\right)^{4}$ in the point-dipole limit. Hence the magnitude of the electrostatic stress must depend on the particle size distribution. Since the stress is also a sensitive function of suspension structure (i.e., the relative positions of all spheres), which is yet to be determined for polydisperse suspensions, further analysis of the effect of polydispersity on the magnitude of the stress is deferred to future studies (Ahn and Klingenberg, 1992).

\section{Effects of particle shape (distribution) and nonlinearity}

In analogy to the discussion of the influence of size polydispersity on the dynamic response, shape polydispersity is expected to affect dispersion. For instance, single- 
particle relaxation occurs for nonspherical particles due to competition between electrostatic and hydrodynamic torques, with the relaxation time dependent upon the degree of shape anisotropy [see for instance Arp et al. (1980)]. In combination with particle interactions, multiple relaxation mechanisms will produce complicated particle dynamics and observed responses. The extent of shape effects is uncertain and awaits further experimental and theoretical investigation.

Another possible source of dispersion broadening arises when the response becomes nonlinear. Otsubo et al. (1992) observed a transition from a pronounced peak in $G^{\prime \prime}(\omega)$ to a broad dispersion as the strain amplitude increased from 0.01 to 0.2 . Nonlinearity in the direct electrostatic stress will arise locally when the strain amplitude is sufficiently large such that the net electrostatic force on a sphere is no longer linear in deformation. Moduli dispersion broadening is expected since the onset of nonlinearity is a local phenomenon and hence varies from place to place in a suspension. Again, however, the relations between nonlinearity, relaxation times, and dispersion are complicated and await further investigation.

\section{E. Effect of accurate hydrodynamic and electrostatic forces}

Thus far, only the direct electrostatic contribution to the shear moduli $\left(G^{\left.E^{\prime} *, G^{E^{\prime \prime}} *\right)}\right.$ has been considered, using a model based on simplified electrostatic and hydrodynamic forces. The electrostatic force on each sphere has been treated as the pairwise summation of the interactions between aligned electric dipoles where the dipole strength is taken as that of an isolated dielectric sphere in an unbounded, uniform external field (point-dipole approximation). This approximation neglects interactions between higher order electric multipoles on neighboring spheres as well as the multibody effect arising from mutual polarization of all spheres; both effects produce a stronger attractive force between sphere pairs as $\alpha=\epsilon_{p} / \epsilon_{c}$ increases (Arp and Mason, 1977; Klingenberg et al., 1991b). The hydrodynamic force on each sphere has also been treated simply, taken as Stokes' drag, ignoring all hydrodynamic interactions between spheres and between each sphere and the planar electrodes. More accurate treatments of the multibody electrostatic and hydrodynamic forces are expected to alter the moduli magnitudes and location of events in the frequency domain presented in this study. Determining whether or not such an effort would significantly alter the qualitative character of the dispersion [e.g., broaden the pronounced peak in $\left.G^{\prime \prime *}\left(\omega^{*}\right)\right]$ must come from a more detailed study.

Finally, the model presented here ignores the direct hydrodynamic stress, $\sigma^{H}$ [Sec. II, Eq. (13)], and its contribution to the moduli $\left(G^{H \prime *}, G^{H \prime *}\right)$. The conditions for which this direct contribution will significantly influence the observed response cannot be determined within the framework of the present investigation, and hence it is uncertain whether dispersion broadening of $G^{\prime \prime *}\left(\omega^{*}\right)$ can be produced by this feature. However, conclusions can be drawn about contributions to the storage modulus. Elasticity $\left(G^{*}\right)$ arises from nonhydrodynamic forces and scales with the interparticle potential (Russel et al., 1989). Marshall et al. (1989) considered the relative importance of colloidal to electrostatic polarization forces in determining the ER behavior of suspensions of poly (methacrylate) particles in a chlorinated hydrocarbon continuous phase. They found that for large particles and large electric field strengths, electrostatic polarization forces dominate over colloidal forces (thermal, van der Waals, and DLVO-type electrostatic forces) in controlling suspension structure and rheological properties. Hence, for large particles under large electric fields, $G^{\prime *}$ is completely 
determined by the electrostatic contribution, $G^{E \prime *}$. Hydrodynamic forces will only influence the transition between the small and large frequency limits. For small particles under small electric fields, the response will be more complicated; the transition from small to large particle/electric field behavior is undetermined at this time.

\section{v. CONCLUSIONS}

The dynamic oscillatory behavior of ER suspensions has been investigated in this study using a molecular dynamics-like simulation technique. This technique employs an idealized model of ER suspensions, consisting of monodisperse suspensions of dielectric spheres whose motion is governed by simplified electrostatic and hydrodynamic forces. Investigation of the relations among forces at the microscopic level, suspension structure, and macroscopic behavior has provided new information about the response of this model system. The significant results of this study, pertaining to such idealized ER suspensions, may be summarized as follows:

(i) for small amplitude deformation of monodisperse suspensions, the direct electrostatic contribution to the dynamic response possesses the property of timeelectric field strength superposition in analogy to time-temperature superposition in polymer rheology;

(ii) a relaxation mechanism arises from competition between electrostatic and hydrodynamic forces acting on spheres within clusters, resulting in a transition in the dynamic structure and rheological response between the small and large frequency limits;

(iii) relaxation occurs over a narrow frequency range for monodisperse suspensions;

(iv) additional features must be included in the model to observe broad dispersion; such features may include particle size or shape polydispersity, or nonlinear deformation; and

(v) for large spheres in large electric fields, the storage modulus is determined completely by the direct electrostatic contribution, $G^{E}$; hydrodynamic forces only influence the transition between the small and large frequency limits.

Each of these predictions can be verified by experiment.

Improving understanding of how microscopic forces and processes influence macroscopic behavior is valuable for making progress in ER suspension and device design. For instance, knowledge of mechanisms facilitates interpretation of experimental results, determining features that produce observed behavior and separating material properties from artifacts. Such knowledge also provides a basis for selection of material properties and suspension parameters for design of desired responses.

Further progress will come from experimental verification of the predictions listed above, determining relations between the response and variables that are known to influence behavior (e.g., particle size distribution), and by extending these concepts to probe more complicating factors in real ER suspensions and applications, such as nonlinear and transient responses, and the role of activators, ionic species, etc.

\section{ACKNOWLEDGMENTS}

The author would like to thank Professor D. E. Brooks of the University of British Columbia for computer time and valuable discussions, and one of the reviewers for insightful comments on the limitations of this work. 


\section{References}

Ahn, K. H. and D. J. Klingenberg, "Relaxation time dispersion in polydisperse electrorheological suspensions," in preparation (1992).

Arp, P. A. and S. G. Mason, "Particle behavior in shear and electric fields VIII. Interactions of pairs of conducting spheres (theoretical)," Coll. Polym. Sci. 255, 566-584 (1977).

Arp, P. A., R. T. Foister, and S. G. Mason, "Some electrohydrodynamic effects in fluid dispersions," Adv. Coll. Interface Sci. 12, 295-356 (1980)

Batchelor, G. K., "The stress system in a suspension of force-free particles," J. Fluid Mech. 41, 545-570 (1970).

Batchelor, G. K., "The effect of Brownian motion on the bulk stress in a suspension of spherical particles," J. Fluid Mech. 83, 97-117 (1977).

Block, H. and J. P. Kelly, "Electro-rheology," J. Phys. D 21, 1661-1677 (1988).

Deinega, Y. F. and G. V. Vinogradov, "Electric fields in the rheology of disperse systems," Rheol. Acta 23, 636-651 (1984).

Ferry, J. D., Viscoelastic Properties of Polymers, 3rd ed. (Wiley, New York, 1980).

Gamato, D. R. and F. E. Filisko, "High frequency dynamic mechanical study of an aluminosilicate electrorheological material," J. Rheol. 35, 1411-1425 (1991).

Gast, A. P. and C. F. Zukoski, "Electrorheological fluids as colloidal suspensions," Adv. Coll. Interface Sci. 30, 153-202 (1989).

Hartsock, D. L., R. F. Novak, and G. J. Chaundy, "ER fluid requirements for automotive devices," J. Rheol. 35, 1305-1326 (1991).

Jordan, T. C. and M. T. Shaw, "Electrorheology," IEEE Trans. Elect. Insul. 24, 849-878 (1989).

Klingenberg, D. J., F. van Swol, and C. F. Zukoski, "Dynamic simulation of electrorheological suspensions," J. Chem. Phys. 91, 7888-7895 (1989).

Klingenherg, D. I., F. van Swol, and C. F. 7.ukoski, "The small shear rate response of electrorheological suspensions: I. Simulation in the point-dipole limit," J. Chem. Phys. 94, 6160-6169 (1991a).

Klingenberg, D. J., F. van Swol, and C. F. Zukoski, "The small shear rate response of electrorheological suspensions: II. Extension beyond the point-dipole limit," J. Chem. Phys. 94, 6170-6178 (1991b).

Klingenberg, D. J., D. Dierking, and C. F. Zukoski, "Stress-transfer mechanisms in electrorheological suspensions," J. Chem. Soc. Faraday Trans. 87, 425-430 (1991c).

Klingenberg, D. J. and C. F. Zukoski, "Studies on the steady-shear behavior of electrorheological suspensions," Langmuir 6, 15-24 (1990).

Korobko, E. V. and Z. P. Shulman, "Viscoelastic behaviour of electrorheological fluids, in Proceedings of the Second International Conference on Electrorheological Fluids, edited by J. D. Carlson, A. F. Sprecher, and H. Conrad (Technomic, Lancaster, PA, 1990), pp. 3-13.

Marshall, L., J. W. Goodwin, and C. F. Zukoski, "Effects of electric fields on the rheology of nonaqueous concentrated suspensions," J. Chem. Soc. Faraday Trans. I 85, 2785-2795 (1989).

McLeish. T. C. B., T. Jordan, and M. T. Shaw, "Viscoelastic response of electrorheological fluids. I. Frequency dependence," J. Rheol. 35, 427-448 (1991).

Otsubo, Y., M. Sekine, and S. Katayama, "Electrorheological properties of silica suspensions," J. Rheol. 36, 479-496 (1992).

Russel, W. B., D. A. Saville, and W. R. Schowalter, Colloidal Dispersions (Cambridge University Press, Cambridge, 1989).

Shulman, Z. P., E. V. Korobko, and Y. G. Yanovskii, "The mechanism of the viscoelastic behaviourkof electrorheological suspensions," J. Non-Newt. Fluid Mech. 33, 181-196 (1989).

Vinogradov, G. V., Z. P. Shulman, Y. G. Yanovskii, B. V. Barancheeva, E. V. Korobko, and I. V. Bukovich, "Viscoelastic behavior of electrorheological suspensions," Inzh.-Fiz. Zhurn. 50, 605-609 (1986).

Winslow, W. M. "Induced fibration of suspensions," J. Appl. Phys. 20, 1137-1140 (1949).

$\mathrm{Xu}, \mathrm{Y} .-\mathrm{Z}$ and $\mathrm{K} .-\mathrm{F}$. Liang, "Electrorheological properties of semiconducting polymer-based suspensions," $\mathbf{J}$ Rheol. 35, 1355-1373 (1991).

Yen, W. S. and P. J. Achorn, "A study of the dynamic behavior of an electrorheological fluid," J. Rheol 35, 1375-1384 (1991). 